\title{
Combined expression of protein disulfide isomerase and endoplasmic reticulum oxidoreductin 1- $\alpha$ is a poor prognostic marker for non-small cell lung cancer
}

\author{
KYOUNG MIN KIM ${ }^{1}$, AE RI AN ${ }^{1}$, HO SUNG PARK ${ }^{1}$, KYU YUN JANG $^{1}$, WOO SUNG MOON ${ }^{1}$, \\ MYOUNG JAE KANG ${ }^{1}$, YONG CHUL LEE ${ }^{2}$, JA HONG KU ${ }^{3}$ and MYOUNG JA CHUNG ${ }^{1,4}$ \\ ${ }^{1}$ Department of Pathology, Chonbuk National University Medical School, \\ Research Institute of Clinical Medicine and Research Institute for Endocrine Sciences of Chonbuk National University, \\ Jeonju, Jeonbuk 54907; Departments of ${ }^{2}$ Internal Medicine and ${ }^{3}$ Thoracic and Cardiovascular Surgery, \\ Chonbuk National University Medical School, Jeonju, Jeonbuk 54896; ${ }^{4}$ Biomedical Research Institute of \\ Chonbuk National University Hospital, Jeonju, Jeonbuk 54907, Republic of Korea
}

Received April 2, 2018; Accepted August 2, 2018

DOI: $10.3892 / 01.2018 .9339$

\begin{abstract}
Protein disulfide isomerase (PDI) is one of the most abundant proteins in the endoplasmic reticulum (ER) and is known as a primary ER resident target of cigarette smoke-induced oxidation. PDI dysfunction triggers unfolded protein response and ER stress. Endoplasmic reticulum oxidoreductin 1- $\alpha$ (ERO1A) is a major regulator of PDI, and recent evidence implicates $\mathrm{PDI}$ and ERO1A as tumor prognostic factors. However, the associated role of PDI and ERO1A and their prognostic impact in non-small cell lung cancers (NSCLCs) remains unknown. The present study investigated the expression of PDI and ERO1A using immunohistochemistry and examined its association with smoking status and their prognostic impact in 198 NSCLCs. PDI and ERO1A expression were observed in 71.2 and $69.2 \%$ of NSCLCs, respectively, and their expressions were significantly associated with each other $(\mathrm{P}<0.001)$. Individual PDI $(\mathrm{P}=0.001)$ and ERO1A $(\mathrm{P}=0.005)$ expression were significantly associated with shorter overall survival (OS) in univariate analysis. PDI expression was significantly associated with never smoking $(\mathrm{P}=0.003)$. PDI expression $(\mathrm{P}<0.001)$ and the co-expression of PDI and ERO1A $(\mathrm{P}<0.001)$ were independent poor prognostic factors for OS in patients with NSCLC in multivariate analysis. Individual expression and co-expression of PDI and
\end{abstract}

Correspondence to: Professor Myoung Ja Chung, Department of Pathology, Chonbuk National University Medical School, Research Institute of Clinical Medicine and Research Institute for Endocrine Sciences of Chonbuk National University, San 2-20 Keumam-Dong Dukjin-gu, Jeonju, Jeonbuk 54907, Republic of Korea E-mail: mjchung@jbnu.ac.kr

Key words: protein disulfide isomerase, endoplasmic reticulum oxidoreductin 1- $\alpha$, non-small cell lung cancer, endoplasmic reticulum, endoplasmic reticulum stress, smoking
ERO1A may be used as novel prognostic indicators of NSCLC outcome.

\section{Introduction}

The endoplasmic reticulum (ER) is the main cellular organelle for post-transcriptional modifications, including folding and assembly of most secretory and membrane proteins $(1,2)$. If cells are exposed to stress conditions such as hypoxia and nutrient deprivation, misfolded or unfolded proteins accumulate in the ER lumen, a condition that has been called 'ER stress' (1). In ER stress conditions, the cells induce an adaptive response known as the 'unfolded protein response (UPR).' UPR activation can enhance cell survival by removing misfolded proteins from the ER via autophagy $(3,4)$. However, prolonged UPR activation can also lead to apoptosis (4).

ER stress is considered to be involved in the pathogenesis of various conditions including diabetes mellitus, neurodegenerative disease, atherosclerosis, inflammation, and cancer $(5,6)$. Lack of oxygen and nutrient are common conditions in cancer, and enhanced proliferation and metabolism of cancer cells result in increased protein production $(3,7)$ this all leads to ER stress and UPR. Many investigators have researched the role of ER stress in cancer. In fact, we reported on the expression of ER stress-related molecules in non-small cell lung cancer (NSCLC) patients (8).

Protein disulfide isomerase (PDI) is one of the most common proteins in the ER; it acts as a molecular chaperone by catalyzing disulfide bond oxidation, reduction, and isomerization $(9,10)$. Disulfide bonds are extremely important for the folding and stability of secretory proteins, which comprise approximately $30 \%$ of all proteins. Therefore, PDI function is essential for cell viability, and PDI dysfunction can lead to UPR and cell death. Recent studies suggest that PDI expression is increased in cancer and it was associated with adverse clinical outcomes in patients with cancers such as glioblastoma, breast carcinoma, and hepatocellular carcinoma 
(HCC) (11-14). However, studies using clinical specimens in NSCLC have not yet been reported.

While catalyzing disulfide bond formation in nascent proteins, the active sites of PDI are reduced (10), and for the reduced PDI to regain catalytic function for disulfide bond formation, the active sites of PDI must be reoxidized (10). Endoplasmic reticulum oxidoreductin 1- $\alpha$ (ERO1A) is an oxidoreductase in the ER (15); by oxidizing PDI, ERO1A acts as a major regulator of PDI (16). Although there are few published papers, ERO1A has also been suggested to be poor prognostic factor for cancer patients (17-20). However, little is known about the prognostic impact of the correlative expression of PDI and ERO1A in NSCLC patients.

Cigarette smoke (CS) is known to be an important cause of lung cancer, but the precise mechanism involved in the development of cancer remains unclear. To explain the mechanism by which smoking causes lung cancer, studies on the relationship between smoking and ER stress and UPR in lung cancer are increasing.

In the current study, we investigated the clinical significance of PDI and ERO1A expression by immunohistochemical staining in NSCLC patients. With further analysis we evaluated the prognostic value of the combined expression of PDI and ERO1A in NSCLC. To understand the mechanism of how smoking is involved in NSCLC pathogenesis, we also investigated the relationship between smoking status and expression of these proteins in NSCLC patients.

\section{Materials and methods}

Patients and follow-up. We studied 198 NSCLC patients who had undergone surgery between 2007 and 2010 at Chonbuk National University Hospital. We reviewed diagnosis and pathologic staging according to the American Joint Committee on Cancer staging system (21) and WHO classification (22), and we obtained the patients' clinical and pathologic information by reviewing medical records. In our group of 198 patients, the male to female ratio was 3.12:1 (150 male and 48 female) and the mean age was 65.2 years (range $47-81$ years) at diagnosis. One hundred and five patients with tumor size of $3 \mathrm{~cm}$ or greater received adjuvant chemotherapy (84 patients; paclitaxel + carboplatin and 21 patients; paclitaxel + cisplatin). We classified smoking status following the Centers for Disease Control and Prevention guidelines of never, former (adults who have smoked at least 100 cigarettes in their lifetime but say they currently do not smoke), or current (adults who have smoked 100 cigarettes in their lifetime and currently smoke cigarettes; (23). This study was approved by the institutional review board of Chonbuk National University Hospital (CUH 2016-05-003-001) and was performed according to Declaration of Helsinki. Based on the retrospective and anonymous character of the study the approval contained a waiver for written informed consent.

Immunohistochemical staining and scoring. Tissue microarrays were established using paraffin-embedded tissues of NSCLC patients. Original H\&E slides were reviewed, and $3.0-\mathrm{mm}$ cores from the representative solid areas were taken. Antigens were retrieved for 12 min in EDTA buffer.
Tissue sections were then incubated overnight with primary antibodies for PDI (1:200, clone RL90; Cell Signaling Technology, Inc., Beverly, MA, USA) and ERO1A (1:200, clone 4G3; Abnova, Taipei, Taiwan). We performed immunohistochemical staining for two antibodies on consecutive slides. The slides that had been stained for PDI and ERO1A were evaluated by two pathologists (Myoung Ja Chung and Kyoung Min Kim) who had no clinicopathologic information on the patients. Expression of both PDI and ERO1A was scored semi-quantitatively by assessing both intensities $(0$, no; 1 , weak; 2 , intermediate; or 3 , strong staining) and proportion of stained area ( 0 , no cell staining; $1,1 \% ; 2,2-10 \% ; 3,11-33 \%$; 4, 34-66\%; and 5, 67-100\%). Intensity and proportion score were summed to obtain final expression scores ranging from zero to eight.

Statistical analysis. The cutoff points for the PDI- and ERO1A-positivity were determined at the point with the highest area under the curve (AUC) to estimate survival of NSCLC patients. Because the ROC curve is a plot of the true positive rate (sensitivity) vs. the false positive rate (1-specificity) for the determination of the death of patients, the cutoff level for the ideal test is presented as the sensitivity 1 and specificity 1 (AUC 1.000). Therefore, we choose the cut-off point at the highest AUC value. The cutoff point for the combined score of PDI (intensity score + proportion score) immunostaining was six. The AUC was 0.615 for PDI. The immunostaining for PDI was scored positive when the combined score was greater than or equal to six. The cutoff point for the combined score of ERO1A immunostaining was five. The AUC was 0.616 for ERO1A. The immunostaining for ERO1A was scored positive when the combined score was greater than or equal to five.

The date of last follow-up was up to last contact or death of patients through August 2016. We evaluated the prognosis of the NSCLC patients by analyzing overall survival (OS), and we considered patient death from lung cancer was considered an event for OS analysis.

We performed all statistical univariate and multivariate Cox proportional hazards regression analyses, Kaplan-Meier survival analysis, and Pearson's Chi-square testing using SPSS software version 19.0 (IBM Corp., Armonk, NY, USA), and $\mathrm{P}<0.05$ was considered to indicate a statistically significant difference.

\section{Results}

Correlation between PDI and EROIA expression and clinicopathologic parameters of NSCLC patients. PDI and ERO1A were predominantly expressed in the cytoplasm of tumor cells (Fig. 1A); non-neoplastic stromal cells did not express either. The cutoffs for the PDI and ERO1A expression scores were six and five, respectively (Fig. 1B). Based on these cutoffs, we classified the expression of PDI and ERO1A as positive in $71.2 \%$ (141 out of 198) and 69.2\% (137 out of 198) of NSCLC patients, respectively.

PDI expression was significantly associated with never smoking $(\mathrm{P}=0.003)$, and ERO1A expression was significantly higher in adenocarcinoma and other cancers than in squamous carcinoma $(\mathrm{P}=0.004)$. In addition, $\mathrm{PDI}$ expression had 
A
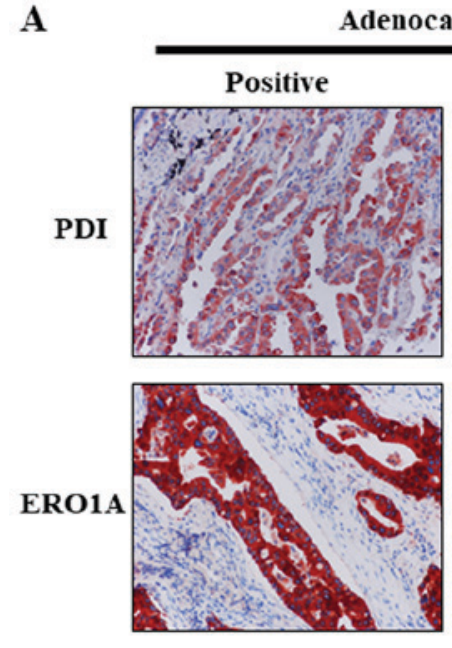

rcinoma
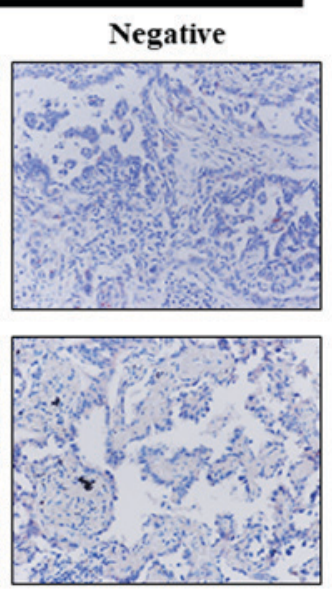

Squamous cell carcinoma
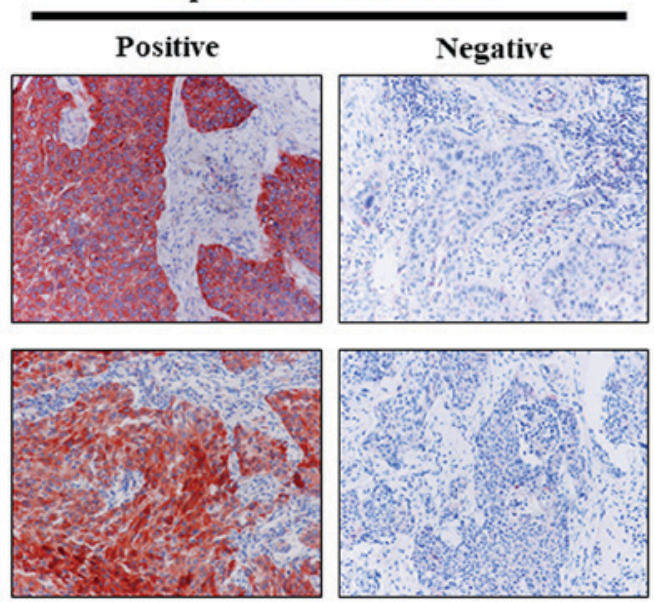

B

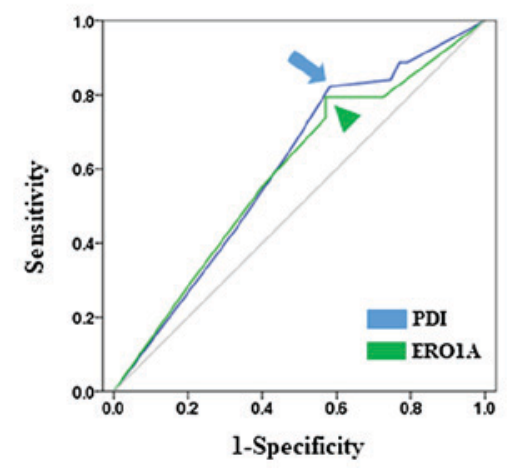

\begin{tabular}{cccc}
\hline & Cut-off & AUC & p \\
\hline PDI & All & 0.598 & 0.017 \\
& $\geq 6$ & 0.615 & 0.005 \\
\multirow{2}{*}{ ERO1A } & All & 0.592 & 0.025 \\
& $\geq 5$ & 0.616 & 0.005 \\
\hline
\end{tabular}

Figure 1. Expression of PDI and ERO1A in 198 NSCLCs. (A) Immunohistochemical expression of PDI and ERO1A in NSCLC. (B) The cut-off points were determined by ROC curve analysis at the highest area under the curve for the estimation overall survival of NGSLC patients. The arrows (PDI) and arrow head (ERO1A) indicate the cut-offs for each immunostaining. AUC, area under the curve. Original magnification of immunohistochemical images; x200. NSCLC, non-small cell lung cancer; ROC, receiver operating characteristic; PDI, protein disulfide isomerase; ERO1A, endoplasmic reticulum oxidoreductin 1- $\alpha$.

a significant positive correlation with ERO1A expression $(\mathrm{P}<0.001$; Table I). Because PDI and ERO1A work together within the ER and there is a significant positive correlation between PDI and ERO1A expressions in NSCLC, we wondered whether the over-expression of both proteins occurs in the same cell. We performed immunohistochemical staining for the PDI and ERO1A on consecutive slides to examine whether the two proteins were co-expressed in tumor cells. In cases showing positivity in both PDI and ERO1A, expression of PDI and ERO1A was co-localized in tumor cells (Fig. 2).

NSCLC patients with PDI and EROIA expression had shorter $O S$ in univariate analysis. Higher tumor stage, PDI expression, and ERO1A expression had significant impact on the OS of NSCLC patients in univariate analysis (Table II). The patients with PDI expression showed 2.215 times greater risk of death from NSCLC [95\% confidence interval (95\% CI): 1.348-3.64, $\mathrm{P}=0.002]$. ERO1A expression predicted a 1.932 times greater risk of NSCLC death (95\% CI: 1.208-3.09, $\mathrm{P}=0.006)$. The survival curves for OS for each marker are presented in Fig. 3.

Due to a significant positive correlation between PDI and ERO1A expressions in NSCLC (Table I), we wonder whether the prediction of NSCLC prognosis was more accurate based on the combined expression of PDI and ERO1A than with the expression of either alone. First, we grouped NSCLC into four-groups according to the expression types of PDI and ERO1A (PDI-/ERO1A-, PDI+/ERO1A-, PDI-/ERO1A+, and PDI+/ERO1A+) and performed a Kaplan-Meier survival analysis (Fig. 3C). Based on these results, we re-grouped the PDI/ERO1A expression pattern into three sub-groups (PDI-/ERO1A-, PDI+/ERO1A- or PDI-/ERO1A+, and PDI+/ERO1A+). We then compared the OS between the three groups by univariate analysis and Kaplan-Meier survival analysis. The type of co-expression pattern (PDI+/ERO1A+) was significantly associated with shorter OS (overall $\mathrm{P}=0.002$ ) by univariate analysis (Table II). The patients with PDI+/ERO1A+ had the poorest prognoses, and the patients with PDI-/ERO1A- had the best (Table II and Fig. 3C). The prognosis of 'PDI+/ERO1A- or PDI-/ERO1At' sub-group was intermediate to the PDI-/ERO1A- and PDI+/ERO1A+ sub-groups (Fig. 3D). The patients with PDI+/ERO1A- or PDI-/ERO1A+ pattern showed a better prognosis than did those with PDI+/ERO1 A+ ( $\mathrm{P}=0.032$; Table II). However, there was no significant difference in OS between PDI-/ERO1Aand PDI+/ERO1A- or PDI-/ERO1A+ (P=0.203).

Multivariate analysis revealed that individual PDI expression and co-expression of PDI and EROIA are independent poor prognostic indicators for NSCLC patients. We included in the multivariate analysis the factors that were significant or borderline significant $(\mathrm{P}<0.1)$ in univariate analysis. We determined current or former smoker, higher tumor stage, and PDI expression to be independent poor prognostic factors for OS in NSCLC patients. Patients with PDI expression had 2.33 times greater risk 
Table I. Association between clinicopathological factors and immunohistochemical expression of PDI and ERO1A in non-small cell lung cancers.

\begin{tabular}{|c|c|c|c|c|c|c|c|c|c|}
\hline \multirow[b]{2}{*}{ Characteristics } & \multirow[b]{2}{*}{ Total } & \multirow{2}{*}{$\frac{\text { PDI }}{\text { Positive }(\%)}$} & \multirow[b]{2}{*}{ P-value } & \multirow{2}{*}{$\frac{\text { ERO1A }}{\text { Positive (\%) }}$} & \multirow[b]{2}{*}{ P-value } & \multicolumn{3}{|c|}{ PDI/ERO1A expression } & \multirow[b]{2}{*}{ P-value } \\
\hline & & & & & & $-/-(\%)$ & $-/+$ or $+/-(\%)$ & $+/+(\%)$ & \\
\hline All cases & 198 & $141(71.2)$ & & 137 (69.2) & & $34(17.2)$ & $50(25.3)$ & $114(57.6)$ & \\
\hline \multicolumn{10}{|l|}{ Sex } \\
\hline Male & 150 & $103(68.7)$ & & $102(68)$ & & $29(19.3)$ & $37(24.7)$ & $84(56)$ & \\
\hline Female & 48 & $38(79.2)$ & 0.162 & $35(72.9)$ & 0.521 & $5(10.4)$ & $13(27.1)$ & $30(62.5)$ & 0.362 \\
\hline \multicolumn{10}{|l|}{ Age (years) } \\
\hline$\leq 65$ & 90 & $62(68.9)$ & & $60(66.7)$ & & $17(18.9)$ & $24(26.7)$ & $49(54.4)$ & \\
\hline$>65$ & 108 & $79(73.1)$ & 0.51 & $77(71.3)$ & 0.482 & $17(15.7)$ & $26(24.1)$ & $65(60.2)$ & 0.706 \\
\hline \multicolumn{10}{|l|}{ Smoking history } \\
\hline Never smoker & 99 & $80(80.8)$ & & $71(71.7)$ & & $11(11.1)$ & $25(25.3)$ & $63(63.6)$ & \\
\hline Smoker & 99 & $61(61.6)$ & 0.003 & $66(66.7)$ & 0.442 & $23(23.2)$ & $25(25.3)$ & $51(51.5)$ & 0.064 \\
\hline \multicolumn{10}{|l|}{ Histologic type } \\
\hline SQCC & 97 & $64(66)$ & & $57(58.8)$ & & $27(27.8)$ & $19(19.6)$ & $51(52.6)$ & \\
\hline $\mathrm{ADC}$ & 94 & $73(77.7)$ & & $73(77.7)$ & & $7(7.4)$ & $28(29.8)$ & $59(62.8)$ & \\
\hline Other & 7 & $4(57.1)$ & 0.144 & $7(100)$ & 0.004 & $0(0)$ & $3(42.9)$ & $4(57.1)$ & 0.002 \\
\hline \multicolumn{10}{|l|}{ Histologic grade } \\
\hline Well or moderate & 159 & $117(73.6)$ & & $112(70.4)$ & & $25(15.7)$ & $39(24.5)$ & $95(59.7)$ & \\
\hline Poor & 39 & $24(61.5)$ & 0.137 & $25(64.1)$ & 0.442 & $9(23.1)$ & $11(28.2)$ & $19(48.7)$ & 0.403 \\
\hline \multicolumn{10}{|l|}{ T stage 8 th } \\
\hline $\mathrm{T} 1,2$ & 154 & $112(72.7)$ & & $106(68.8)$ & & $26(16.9)$ & $38(24.7)$ & $90(58.4)$ & \\
\hline $\mathrm{T} 3,4$ & 44 & $29(65.9)$ & 0.378 & $31(70.5)$ & 0.837 & 8 (18.2) & $12(27.3)$ & $24(54.5)$ & 0.898 \\
\hline \multicolumn{10}{|l|}{$\mathrm{N}$ stage } \\
\hline No & 134 & 100 (74.6) & & $92(68.7)$ & & $19(14.2)$ & $38(28.4)$ & $77(57.5)$ & \\
\hline N 1-3 & 64 & $41(64.1)$ & 0.125 & $45(70.3)$ & 0.813 & $15(23.4)$ & $12(18.8)$ & $37(57.8)$ & 0.154 \\
\hline \multicolumn{10}{|l|}{ PDI } \\
\hline Negative & 57 & & & $23(40.4)$ & & & & & \\
\hline Positive & 141 & & & $114(80.9)$ & $<0.001$ & & & & \\
\hline \multicolumn{10}{|l|}{ ERO1A } \\
\hline Negative & 61 & $27(44.3)$ & & & & & & & \\
\hline Positive & 137 & 114 (83.2) & $<0.001$ & & & & & & \\
\hline
\end{tabular}

Smoker, current or former; SQCC, squamous cell carcinoma; ADC, adenocarcinoma; PDI, protein disulfide isomerase; ERO1A, endoplasmic reticulum oxidoreductin $1-\alpha$.

of death from NSCLC (95\% CI: 1.322-4.105, P=0.003; model 1, Table III). Additionally, we inserted the combined PDI/ERO1A expression pattern instead of individual expression of PDI and ERO1A in multivariate analysis. Current or former smoker, higher tumor stage, and co-expression of PDI+/ERO1A+ were independent poor prognostic indicators for OS in NSCLC patients, patients with co-expression of PDI+/ERO1A+ showed a 3.517 times greater risk of death from NSCLC (95\% CI: 1.736-7.122, $\mathrm{P}<0.001)$ than did the patients who showed PDI-/ERO1A- expression pattern (model 2, Table III).

\section{Discussion}

In the present study, we investigated immunohistochemical expression of PDI and ERO1A in human NSCLC tissues. To the best of our knowledge, this is the first study of the correlative expression of PDI and ERO1A in NSCLC. Our results show: i) expression of PDI and ERO1A in 71.2 and $69.2 \%$ of NSCLC, respectively; ii) significant positive correlation $(\mathrm{P}<0.001)$ for expression of PDI and ERO1A; iii) significant association of individual and co-expression PDI and ERO1A with shorter OS in univariate and Kaplan-Meier survival analysis; and iv) poor independent prognosis value of individual PDI expression and $\mathrm{PDI}+/ \mathrm{ERO} 1 \mathrm{~A}+$ co-expression for OS in NSCLC patients in multivariate analysis.

PDI is a $57-\mathrm{kDa}$ sized molecular chaperone and one of the most abundant proteins in the ER (9). The main function of PDI is to catalyze disulfide bond formation, breakage, and rearrangement of its protein and peptide substrates. Disulfide bonds are formed between the sulfhydryl cysteine groups and 
Table II. Univariate Cox regression analysis for the overall survival of patients with non-small cell lung cancer.

\begin{tabular}{|c|c|c|c|}
\hline Variables & Risk ratio & $95 \%$ confidence interval & P-value \\
\hline Sex, female (vs. male) & 0.634 & $0.389-1.031$ & 0.066 \\
\hline Age, $>65($ vs. $\leq 65)$ & 1.436 & $0.974-2.118$ & 0.068 \\
\hline Smoking history, smoker (vs. never smoker) & 1.402 & $0.955-2.059$ & 0.085 \\
\hline Histologic grade, poor (vs. well or moderate) & 0.607 & $0.539-1.436$ & 0.607 \\
\hline T stage, T 3,4 (vs. T 1,2) & 1.630 & $1.062-2.503$ & 0.025 \\
\hline N stage, N 1,2 (vs. N 0) & 1.203 & $0.802-1.805$ & 0.372 \\
\hline PDI expression, positive (vs. negative) & 2.215 & $1.348-3.640$ & 0.002 \\
\hline ERO1A expression, positive (vs. negative) & 1.932 & $1.208-3.090$ & 0.006 \\
\hline PDI/ERO1A expression pattern, -/- & 1 & & \\
\hline$-/+$ or $+/-$ & 1.634 & $0.767-3.480$ & 0.203 \\
\hline$+/+$ & 2.798 & $1.445-5.417$ & 0.002 \\
\hline PDI/ERO1A expression pattern, -/+ or +/- & 1 & & \\
\hline$+/+$ & 1.702 & $1.047-2.767$ & 0.032 \\
\hline
\end{tabular}

Smoker, current or former; PDI, protein disulfide isomerase; ERO1A, endoplasmic reticulum oxidoreductin 1- $\alpha$.
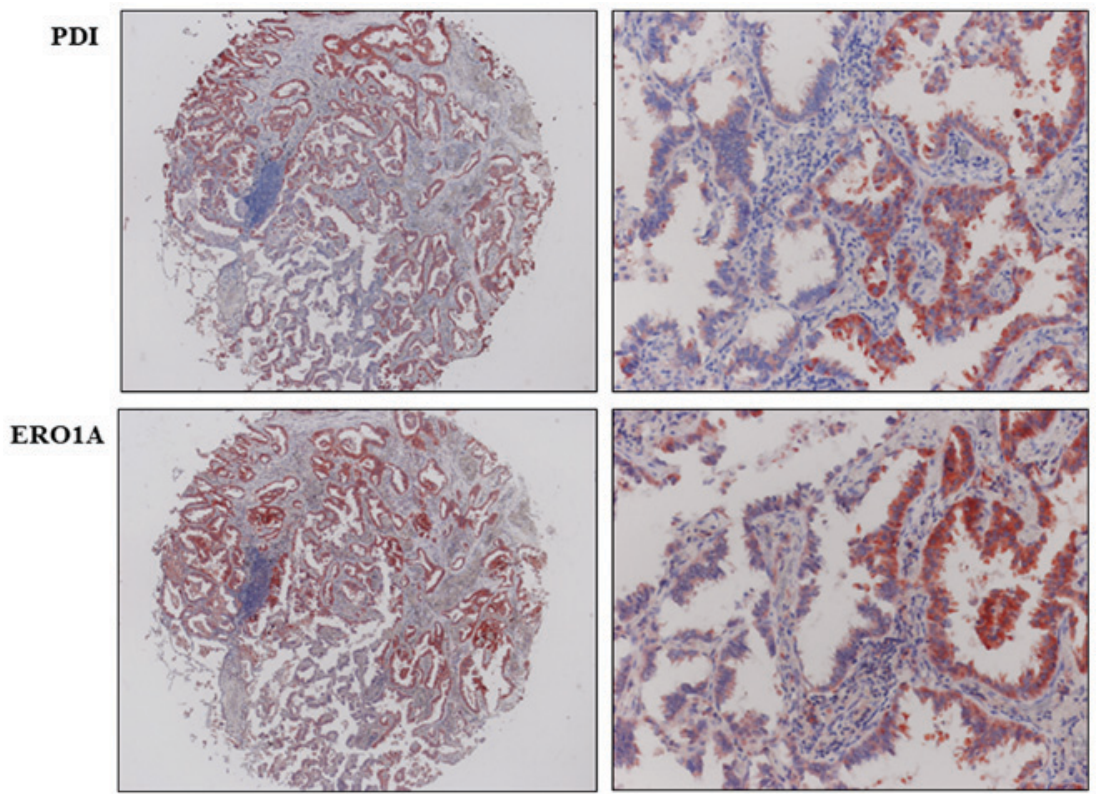

Figure 2. Immunohistochemical staining for PDI and ERO1A on consecutive slides in lung adenocarcinoma. The two antibodies were found to have a very similar staining pattern. Expression of PDI and ERO1A were found to be co-localized in tumor cells. Original magnification of immunohistochemical images; x40 in left side images, x200 in right side images. PDI, protein disulfide isomerase; ERO1A, endoplasmic reticulum oxidoreductin 1- $\alpha$.

are involved in the stabilization of the protein tertiary structure which is essential for the proteins' function (10). Therefore, PDI dysfunction results in accumulation of unfolded and misfolded proteins in the ER. Aberrant PDI expression or function is reported to be involved in many human diseases such as neurodegenerative (24) and cardiovascular diseases (25) and also in cancer (10).

PDI expression was significantly higher in various cancer types including lung cancer (10) and was associated with adverse clinical outcomes in various cancer types (11-14). Functional studies also indicate that PDI is involved in cancer progression and cancer cell survival (26-28). There are several proposed mechanisms to explain the relevance of PDI over-expression and adverse clinical outcomes. First, PDI over-expression may protect cancer cells from apoptosis. Inhibiting PDI increased chemotherapy-induced apoptosis in melanoma (26). A PDI inhibitor induced apoptosis in HCC cells and ovarian cancer cells (10). Second, PDI is suggested to be associated with neutralization of the transforming growth factor- $\beta 1$ (TGF- $\beta 1$ ) effect. TGF- $\beta 1$ is a strong cell proliferation inhibitor and frequently inhibits cancer growth (27). Lastly, PDI can affect cancer invasion and metastasis. Matrix metallopeptidase 9 (MMP-9) is an important protein for cancer cell metastasis (28). PDI-mediated 
Table III. Multivariate Cox regression analysis for the overall survival of patients with non-small cell lung cancer.

\begin{tabular}{|c|c|c|c|}
\hline Variables & Risk ratio & $95 \%$ confidence interval & P-value \\
\hline \multicolumn{4}{|l|}{ Model $1^{\mathrm{a}}$} \\
\hline Smoking, smoker (vs. never smoker) & 1.594 & $1.063-2.388$ & 0.024 \\
\hline T stage, T 3,4 (vs. T 1,2) & 1.862 & $1.194-2.903$ & 0.006 \\
\hline PDI expression, positive (vs. negative) & 2.33 & $1.322-4.105$ & 0.003 \\
\hline \multicolumn{4}{|l|}{ Model $2^{\mathrm{b}}$} \\
\hline Smoking, smoker (vs. never smoker) & 1.536 & $1.031-2.288$ & 0.035 \\
\hline T stage, T 3,4 (vs. T 1,2) & 1.831 & $1.174-2.854$ & 0.008 \\
\hline PDI/ERO1A expression, -/- & 1 & & $<0.001$ \\
\hline$-/+$ or $+/-$ & 1.81 & 0.82-3.999 & 0.142 \\
\hline$+/+$ & 3.517 & $1.736-7.122$ & $<0.001$ \\
\hline
\end{tabular}

Smoker, current or former; PDI, protein disulfide isomerase; ERO1A, endoplasmic reticulum oxidoreductin 1- $\alpha .{ }^{a}$ Variables considered in model 1 were sex, age, smoking history, T stage, PDI expression, and ERO1L expression. ${ }^{b}$ Variables considered in model 2 were sex, age, smoking history, T stage, and co-expression of PDI and ERO1L.
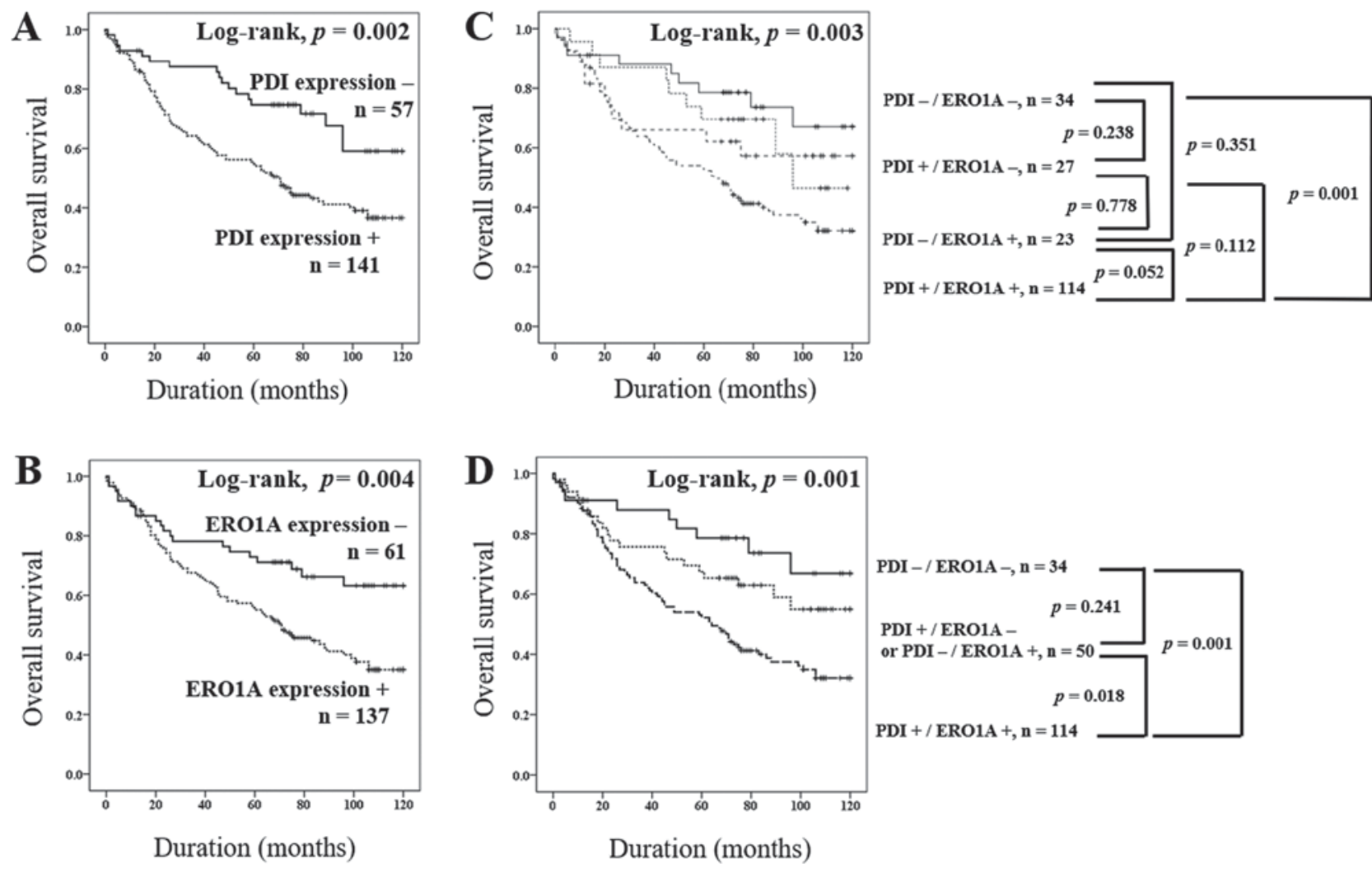

Figure 3. Prognostic significance of PDI and ERO1A in 198 non-small cell lung cancers; Kaplan-Meier survival analysis for overall survival according to the individual (A) PDI and (B) ERO1A, and combined expression of PDI and ERO1A (C) 4 groups and (D) 3 groups, in NSCLC patients.

disulfide bond formation is essential for MMP secretion and gelatinolytic activity. Our result showed that PDI expression was associated with adverse OS in NSCLC patients on univariate and Kaplan-Meier survival analysis and was an independent poor prognostic indicator in NSCLC patients on multivariate Cox regression analysis. Our results do not explain the mechanism of the relationship between PDI over-expression and short OS. In previous reports, however, it was suggested that PDI protects tumor cells from oxidative damage in (10). Therefore, we assume that cancer cells with PDI over-expression can be protected from apoptosis and survival can be promoted, which may explain the relationship between PDI over-expression and poor prognosis. Additional studies are needed to clarify the role of PDI over-expression in poor NSCLC prognosis.

Exposure to CS is a major risk factor for the development of lung cancer. A previous study reported that PDI was up-regulated in smokers, and it is a primary ER-resident 
target of smoking-induced oxidation (29). However, another study showed that smoking-induced radicals can lead to PDI unfolding, inhibiting PDI activity (30). We wondered how smoking status in patients with NSCLC might be related to PDI expression. Therefore, we evaluated the relationship between smoking history and PDI expression, and expression was significantly higher in never smokers than in former or current smokers $(\mathrm{P}=0.003)$. In this study, we cannot explain the mechanism for the relationship between non-smokers and high PDI expression, but based on the results of other researchers, we have inferred some possibilities. CS exposure leads to post-translational modifications and changes in the $3 \mathrm{D}$ structure of PDI and then render it inactive (30). Therefore, PDI over-expression may be less relevant to the pathogenesis of NSCLC in smokers given that smoking can impair PDI generation and function. Other investigators reported that UPR-related proteins (eIF2 $\alpha$, phospho-eIF $2 \alpha$, and BiP) were increased in NSCLC but not directly related to smoking exposure, and these were the common traits observed in lung cancer, regardless of the triggering factors (31). We believe that the association between never smoking and PDI over-expression in our study should be understood through additional studies.

ERO1 is one of the essential proteins for protein disulfide bond formation in the ER in cooperation with PDI. While PDI plays a role in protein folding and isomerization, it takes a reduced form and is then re-oxidized so regulators can oxidize PDI efficiently. ERO1A is known as a major regulator in PDI oxidation. Recent reports have suggested that ERO1A was over-expressed in cancer and had an oncogenic role in cancer (22-25). ERO1A knock-down suppressed the proliferation, migration, invasiveness, and chemoresistance in gastric cancer cells through controlling the phosphorylation state of Akt and JNK. In breast cancer cells, ERO1A promoted immune escape by increasing PD-L1 expression (20). Additionally, ERO1A can play an important role in vascular endothelial growth factor secretion and contributes the neovascular formation of cancer (32). ERO1A over-expression was associated with poor OS among lung adenocarcinoma patients (33). In line with previous reports, our results also show that NSCLC patients with positive ERO1A expression had significantly shorter OS than did patients with negative ERO1A expression. However, more studies are needed to identify the mechanisms that explain the association between ERO1A over-expression and poor prognosis in NSCLC.

Many functional studies have shown that ERO1A is an important regulator of PDI and that the dysregulation of these two proteins is involved in the progression of cancer. However, to our best knowledge, no study authors to date have investigated the correlative role of PDI and ERO1A expression using clinical cancer samples. Our data revealed that the expression of PDI and ERO1A were very significantly $(\mathrm{P}<0.001)$ related. The patient group with co-expression of PDI+/ERO1A+ showed the shortest OS than did the PDI-/ERO1A-group in Kaplan Meier and univariate survival analysis $(\mathrm{P}<0.05)$. The multivariate analysis revealed that PDI+/ERO1A+ were an independent poor prognostic indicator of OS in NSCLC patients. Our results support the previous findings that the PDI and ERO1A are closely related with each other functionally and that the co-expression of PDI and ERO1A can be utilized as a stronger prognostic indicator than the single expression of either alone. However, little is known about the prognostic effect of the combined expression of PDI and ERO1A in cancer patients; given our small sample, a definitive conclusion on the prognosis of NSCLC patients would be premature. In order for PDI and/or ERO1A expression to be used as an important prognostic indicator for patients with NSCLC, we believe that confirmation is necessary through large-scale studies and accumulated results. Modalities of treatment also affect the OS in NSCLC patients. However, the information on the postoperative treatment of the patients was not included in the OS analysis in the present study and we think it is a limitation of this study. Additional studies supplementing this may be helpful in confirming the role as a prognostic marker of PDI and/or ERO1A expression.

In conclusion, this study has demonstrated that the immunohistochemical expression of PDI and ERO1A might be helpful for predicting the prognosis of NSCLC patients.

\section{Acknowledgements}

The biospecimens for this study were provided by the Chonbuk National University Hospital Biobank, a member of the Korea Biobank Network, which is supported by the Ministry of Health, Welfare and Family Affairs, Republic of Korea. All samples derived from the National Biobank of Korea were obtained with informed consent under institutional review board-approved protocols.

\section{Funding}

The present study was supported by the Biomedical Research Institute, Chonbuk National University Hospital.

\section{Availability of data and materials}

The datasets used and/or analyzed during the current study are available from the corresponding author on reasonable request.

\section{Authors' contributions}

KMK, HSP, KYJ, WSM, MJK, YCL, JHK and MJC participated in the study design. KMK, ARA, HSP, KYJ, WSM, MJK and MJC performed the experiments and interpreted the patient data from immunohistochemistry. YCL and JHK organized and analyzed the patient's clinical data. ARA, KMK, HSP, KYJ, WSM, MJK, YCL, JHK and MJC were involved in drafting the manuscript. KMK, ARA, HSP, KYJ, WSM, MJK, YCL, JHK and MJC revised the manuscript critically for important intellectual content and provided final approval of the version to be published. All authors read and approved the final manuscript.

\section{Ethics approval and consent to participate}

The present study was approved by the institutional Review Board of Chonbuk National University Hospital (approval no. CUH 2016-05-003-001) and was performed according to the Declaration of Helsinki. Based on the retrospective and anonymous character of the study the approval contained a waiver for written informed consent. 


\section{Patient consent for publication}

Based on the retrospective, anonymous character of the study, the IRB approval contained a waiver for written informed consent.

\section{Competing interests}

The authors declare that they have no competing interests.

\section{References}

1. Lee AS and Hendershot LM: ER stress and cancer. Cancer Biol Ther 5: 721-722, 2006.

2. Hoseki J, Ushioda R and Nagata K: Mechanism and components of endoplasmic reticulum-associated degradation. J Biochem 147 19-25, 2010.

3. Alasiri G, Fan LY, Zona S, Goldsbrough IG, Ke HL, Auner HW and Lam EW: ER stress and cancer: The FOXO forkhead transcription factor link. Mol Cell Endocrinol 462: 67-81, 2018.

4. Rashid HO, Yadav RK, Kim HR and Chae HJ: ER stress: Autophagy induction, inhibition and selection. Autophagy 11: 1956-1977, 2015.

5. Yoshida H: ER stress and diseases. FEBS J 274: 630-658, 2007.

6. Hosoi T and Ozawa K: Endoplasmic reticulum stress in disease: Mechanisms and therapeutic opportunities. Clin Sci (Lond) 118: 19-29, 2009.

7. Ozcan U, Ozcan L, Yilmaz E, Düvel K, Sahin M, Manning BD and Hotamisligil GS: Loss of the tuberous sclerosis complex tumor suppressors triggers the unfolded protein response to regulate insulin signaling and apoptosis. Mol Cell 29: 541-551, 2008.

8. Kim KM, Yu TK, Chu HH, Park HS, Jang KY, Moon WS, Kang MJ, Lee DG, Kim MH, Lee JH and Chung MJ: Expression of ER stress and autophagy-related molecules in human non-smal cell lung cancer and premalignant lesions. Int J Cancer 131: E362-E370, 2012.

9. Ferrari DM and Söling HD: The protein disulphide-isomerase family: Unravelling a string of folds. Biochem J 339: 1-10, 1999.

10. Xu S, Sankar S and Neamati N: Protein disulfide isomerase: A promising target for cancer therapy. Drug Discov Today 19: 222-240, 2014.

11. Cancer Genome Atlas Research Network: Comprehensive genomic characterization defines human glioblastoma genes and core pathways. Nature 455: 1061-1068, 2008.

12. Shai R, Shi T, Kremen TJ, Horvath S, Liau LM, Cloughesy TF, Mischel PS and Nelson SF: Gene expression profiling identifies molecular subtypes of gliomas. Oncogene 22: 4918-4923, 2003.

13. Thongwatchara P, Promwikorn W, Srisomsap C, Chokchaichamnankit D, Boonyaphiphat $\mathrm{P}$ and Thongsuksai $\mathrm{P}$ : Differential protein expression in primary breast cancer and matched axillary node metastasis.Oncol Rep26: 185-191,2011.

14. Xia W, Zhuang J, Wang G, Ni J, Wang J and Ye Y: P4HB promotes HCC tumorigenesis through downregulation of GRP78 and subsequent upregulation of epithelial-to-mesenchymal transition. Oncotarget 8: 8512-8521, 2017.

15. Sevier CS and Kaiser CA: Erol and redox homeostasis in the endoplasmic reticulum. Biochim Biophys Acta 1783: 549-556, 2008.

16. Araki $\mathrm{K}$ and Nagata K: Functional in vitro analysis of the ERO1 protein and protein-disulfide isomerase pathway. J Biol Chem 286: 32705-32712, 2011.

17. Zhou B, Wang G, Gao S, Chen Y, Jin C, Wang Z, Yang Y, Ma Z, Zhang $\mathrm{W}$ and Feng X: Expression of ERO1L in gastric cancer and its association with patient prognosis. Exp Ther Med 14: 2298-2302, 2017.

18. Seol SY, Kim C, Lim JY, Yoon SO, Hong SW, Kim JW, Choi SH and Cho JY: Overexpression of endoplasmic reticulum oxidoreductin 1- $\alpha$ (ERO1L) is associated with poor prognosis of gastric cancer. Cancer Res Treat 48: 1196-1209, 2016.
19. Kutomi G, Tamura Y, Tanaka T, Kajiwara T, Kukita K, Ohmura T, Shima H, Takamaru T, Satomi F, Suzuki Y, et al: Human endoplasmic reticulum oxidoreductin 1- $\alpha$ is a novel predictor for poor prognosis of breast cancer. Cancer Sci 104: 1091-1096, 2013.

20. Tanaka T, Kutomi G, Kajiwara T, Kukita K, Kochin V, Kanaseki T, Tsukahara T, Hirohashi Y, Torigoe T, Okamoto Y, et al: Cancer-associated oxidoreductase ERO1- $\alpha$ promotes immune escape through up-regulation of PD-L1 in human breast cancer. Oncotarget 8: 24706-24718, 2017.

21. Amin MB, Edge S, Greene F, Byrd DR, Brookland RK, Washington MK, Gershenwald JE, Compton CC, Hess KR, Sullivan DC, et al: AJCC cancer staging manual. 8th edition. Springer International Publishing, New York, NY, 2017.

22. Travis WD, Brambilla E, Burke A, Marx A and Nicholson AG: WHO classification of tumours of the lung, pleura, thymus and heart. 4th edition. IARC press, Lyon, 2015.

23. Schoenborn CA and Adams PE: Health behaviors of adults: United States, 2005-2007. Vital Health Stat 10: 1-132, 2010.

24. Uehara T, Nakamura T, Yao D, Shi ZQ, Gu Z, Ma Y, Masliah E, Nomura Y and Lipton SA: S-nitrosylated protein-disulphide isomerase links protein misfolding to neurodegeneration. Nature 441: 513-517, 2006.

25. Severino A, Campioni M, Straino S, Salloum FN, Schmidt N, Herbrand U, Frede S, Toietta G, Di Rocco G, Bussani R, et al: Identification of protein disulfide isomerase as a cardiomyocyte survival factor in ischemic cardiomyopathy. J Am Coll Cardiol 50: 1029-1037, 2007.

26. Lovat PE, Corazzari M, Armstrong JL, Martin S, Pagliarini V, Hill D, Brown AM, Piacentini M, Birch-Machin MA and Redfern CP: Increasing melanoma cell death using inhibitors of protein disulfide isomerases to abrogate survival responses to endoplasmic reticulum stress. Cancer Res 68: 5363-5369, 2008.

27. Sipes NJ, Miller DA, Bascom CC, Winkler JK, Matrisian LM and Moses HL: Altered regulation of protein disulfide isomerase in cells resistant to the growth-inhibitory effects of transforming growth factor beta 1. Cell Growth Differ 1: 241-246, 1990.

28. Khan MM, Simizu S, Suzuki T, Masuda A, Kawatani M, Muroi M, Dohmae $\mathrm{N}$ and Osada $\mathrm{H}$ : Protein disulfide isomerase-mediated disulfide bonds regulate the gelatinolytic activity and secretion of matrix metalloproteinase-9. Exp Cell Res 318: 904-914, 2012.

29. Kenche H, Ye ZW, Vedagiri K, Richards DM, Gao XH, Tew KD, Townsend DM and Blumental-Perry A: Adverse outcomes associated with cigarette smoke radicals related to damage to protein-disulfide isomerase. J Biol Chem 291: 4763-4778, 2016.

30. Kenche H, Baty CJ, Vedagiri K, Shapiro SD and Blumental-Perry A: Cigarette smoking affects oxidative protein folding in endoplasmic reticulum by modifying protein disulfide isomerase. FASEB J 27: 965-977, 2013.

31. Jorgensen E, Stinson A, Shan L, Yang J, Gietl D and Albino AP: Cigarette smoke induces endoplasmic reticulum stress and the unfolded protein response in normal and malignant human lung cells. BMC Cancer 8: 229, 2008.

32. May D, Itin A, Gal O, Kalinski H, Feinstein E and Keshet E: Ero1-L alpha plays a key role in a HIF-1-mediated pathway to improve disulfide bond formation and VEGF secretion under hypoxia: Implication for cancer. Oncogene 24: 1011-1020, 2005.

33. Hsu CH, Hsu CW, Hsueh C, Wang CL, Wu YC, Wu CC, Liu CC, Yu JS, Chang YS and Yu CJ: Identification and characterization of potential biomarkers by quantitative tissue proteomics of primary lung adenocarcinoma. Mol Cell Proteomics 15: 2396-2410, 2016.

This work is licensed under a Creative Commons Attribution-NonCommercial-NoDerivatives 4.0 International (CC BY-NC-ND 4.0) License. 